\title{
Water-Polymer Coupling Induces a Dynamical Transition in Microgels
}

\author{
Letizia Tavagnacco, ${ }^{\dagger}$ Ester Chiessi, ${ }^{*}{ }^{\ddagger}$ ¿ Marco Zanatta, ${ }^{\text {II }}$ Andrea Orecchini, ${ }^{\S}, \|$ \\ and Emanuela Zaccarelli*,†(i)
}

${ }^{\dagger}$ CNR-ISC and Department of Physics, Sapienza University of Rome, Piazzale A. Moro 2, 00185 Rome, Italy

${ }^{\ddagger}$ Department of Chemical Sciences and Technologies, University of Rome Tor Vergata, Via della Ricerca Scientica I, 00133 Rome, Italy

${ }^{\mathbb{I}}$ Department of Computer Science, University of Verona, Strada Le Grazie 15, 37138 Verona, Italy

${ }^{\S}$ Department of Physics and Geology, University of Perugia, Via A. Pascoli, 06123 Perugia, Italy

"CNR-IOM c/o Department of Physics and Geology, University of Perugia, Via A. Pascoli, 06123 Perugia, Italy

\section{Supporting Information}

\begin{abstract}
The long debated protein dynamical transition was recently found also in nonbiological macromolecules, such as poly- $N$-isopropylacrylamide (PNIPAM) microgels. Here, by using atomistic molecular dynamics simulations, we report a description of the molecular origin of the dynamical transition in these systems. We show that PNIPAM and water dynamics below the dynamical transition temperature $T_{d}$ are dominated by methyl group rotations and hydrogen bonding, respectively. By comparing with bulk water, we unambiguously identify PNIPAM-water hydrogen bonding as mainly responsible for the occurrence of the transition. The observed phenomenology thus crucially depends on the water-macromolecule coupling, being relevant to a wide class of hydrated systems, independently from the biological function.
\end{abstract}

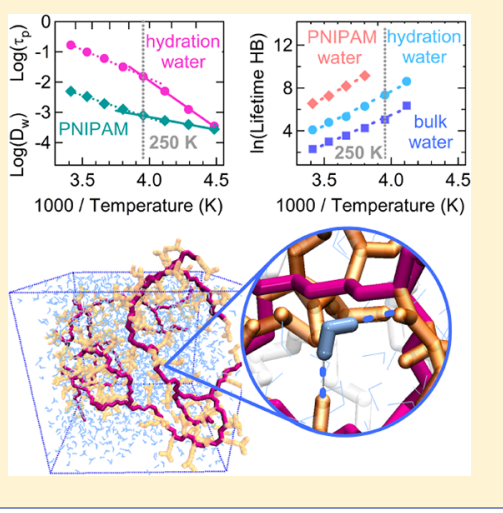

$\mathrm{T}$ he well-known protein dynamical transition takes place in hydrated protein suspensions at a relatively low temperature, usually called $T_{d}$, typically between 220 and $240 \mathrm{~K}$, but depending on the specific system. After the first observation in 1989 for myoglobin, ${ }^{1}$ the transition has been reported in several kinds of proteins, including ribonuclease $\mathrm{A},{ }^{2}$ cytochrome $\mathrm{c}^{3}{ }^{3}$ lysozyme, ${ }^{4}$ and other biomacromolecules such as RNA, ${ }^{5}$ DNA, $^{6}$ and lipid bilayers, ${ }^{7}$ irrespective of secondary structure, folding, and degree of polymerization. ${ }^{8-10}$ The transition consists of a steep enhancement of the atomic mobility that, below $T_{d}$, is limited to harmonic vibrations and methyl rotations. At $T_{d}$, anharmonic motions and local diffusion of groups of atoms are triggered, which, for proteins, is a prerequisite to the onset of activity. ${ }^{11-13}$ It is important to remark that $T_{d}$ does not coincide with the calorimetric glass transition temperature, which is found to occur at even lower temperatures. While the dynamical transition always occurs in aqueous environments, with a water content that is kept to a minimum to avoid the onset of ice crystallization, the role of water is still a debated issue. Evidence was reported in favor of a concomitant activation of the water dynamics at the transition, as shown for folded and intrinsically disordered proteins. $^{10,14,15}$ These studies suggest a strong interplay between protein and water, but different interpretations have also been proposed in the literature going from a water-slaved to a water-driven protein dynamics. ${ }^{16-18}$
Very recently, elastic incoherent neutron scattering experiments reported the occurrence of a dynamical transition at $T_{d}$ $\sim 250 \mathrm{~K}$ also for a nonbiological system, i.e., $\operatorname{poly}(\mathrm{N}$ isopropylacrylamide), PNIPAM, microgels. ${ }^{19}$ Like proteins, PNIPAM microgels have an extended covalent connectivity and an amphipilic character. In addition, their network structure maximizes the ability of water confinement. For the same macromolecular concentration, water accessible surface area in PNIPAM microgels is about 30\% larger as compared to a globular protein, thus magnifying the matrix-induced effect on water properties. ${ }^{20}$ This results in the observation of the dynamical transition in PNIPAM-water suspensions with a very large water content, up to roughly $60 \%$ of water $(\mathrm{w} / \mathrm{w})$. These findings thus extend the dynamical transition concept beyond the world of biological function, encompassing the much wider context of hydrated complex macromolecular entities.

To unveil the molecular mechanisms involved in the dynamical transition, in this work we perform atomistic molecular dynamics simulations using a nanoscale model of the microgel in water (Figure 1A) that quantitatively reproduces the experimental results. ${ }^{19}$ By varying PNIPAM

Received: January 22, 2019

Accepted: February 8, 2019

Published: February 8, 2019 


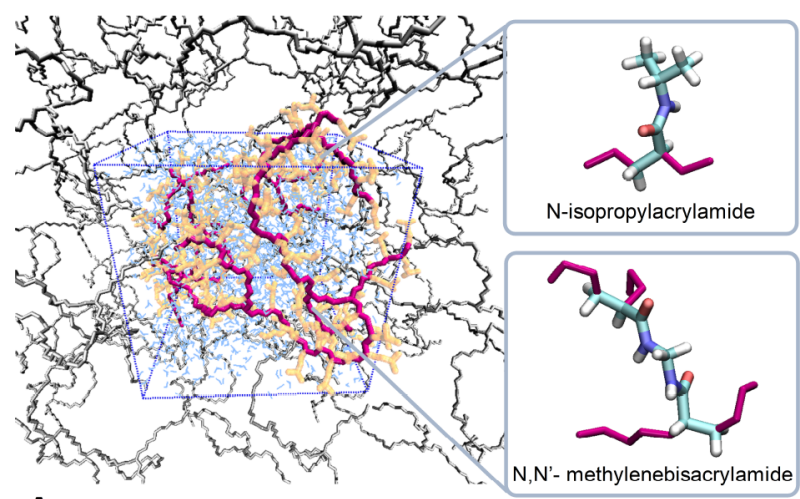

A

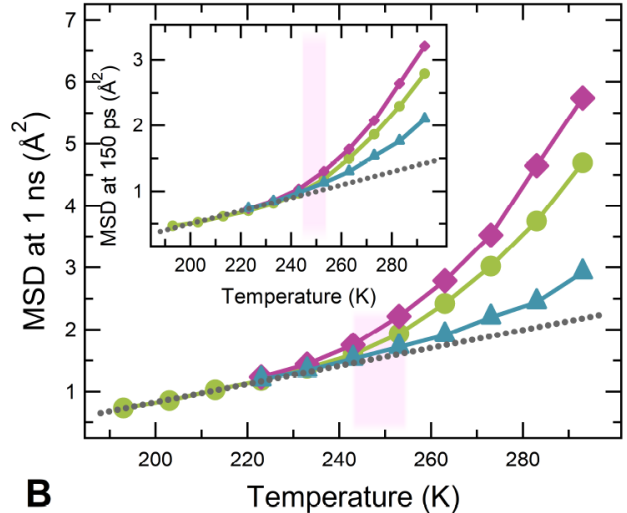

B Temperature $(\mathrm{K})$

Figure 1. (A) Microgel network model. Backbone and side chain atoms are displayed in magenta and yellow, respectively; water molecules and periodic images of backbone atoms are represented in blue and gray, respectively. Polymer hydrogen atoms are omitted for clarity. The two side frames report the chemical structure of the repeating unit (top) and of the cross-link (bottom). (B) Temperature dependence of MSD calculated for PNIPAM hydrogen atoms at $1 \mathrm{~ns}$ (main figure) and 150 ps (inset). Results are displayed for PNIPAM mass fractions of $30 \%$ (purple diamonds), $40 \%$ (green circles), and 60\% (blue triangles). The dashed lines are guides to the eye suggesting a linear behavior corresponding to the dry sample, for which the dynamical transition is suppressed. ${ }^{19}$

A
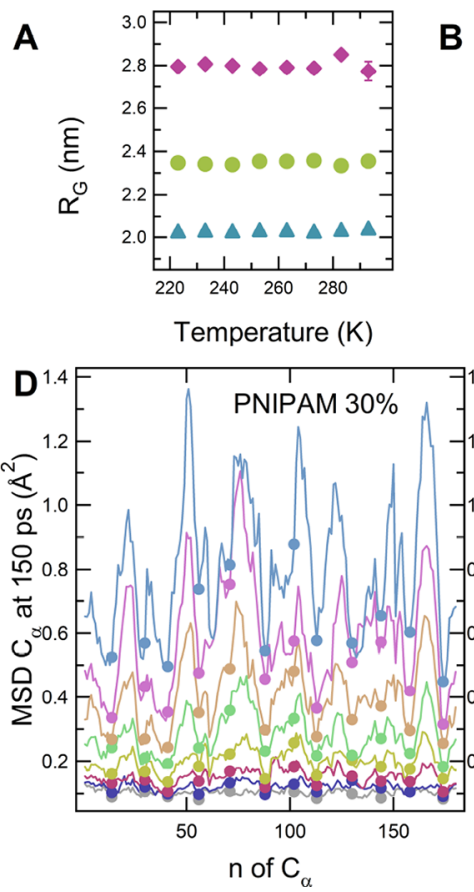

B

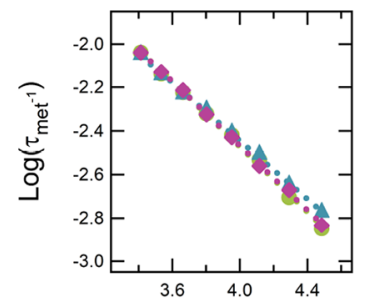

1000 / Temperature (K)
C

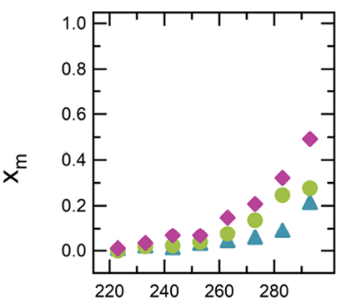

Temperature (K)

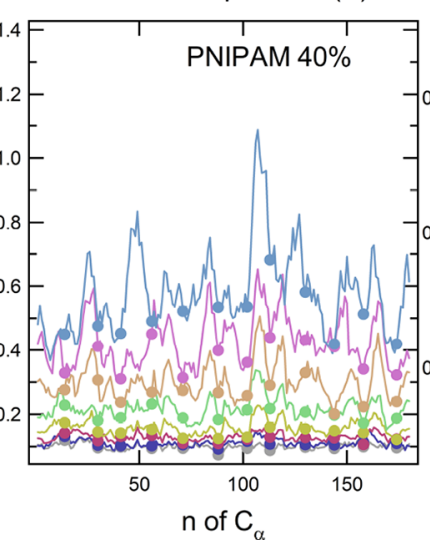

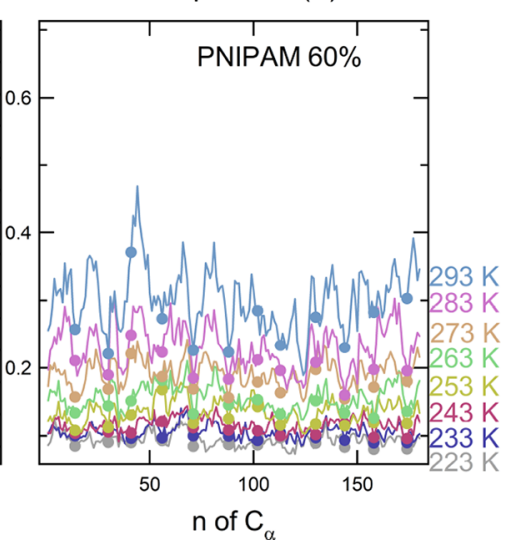

Figure 2. (A) Temperature dependence of PNIPAM radius of gyration. (B) Arrhenius plot of the average lifetime (in ps) of a rotational state, $\tau_{m e t}$ of a methyl group. (C) Fraction of backbone mobile dihedrals as a function of the temperature. In (A), (B), and (C) systems with PNIPAM mass fractions of 30, 40, and $60 \%(\mathrm{w} / \mathrm{w})$ are displayed with purple diamonds, green circles, and blue triangles, respectively. (D) Temperature dependence of the MSD of backbone tertiary carbon atoms $C_{\alpha}$ that are directly connected to the side chain. Here, $n$ is the index of residue, varying from 1 to 180, the total number of repeating units in our network model. Circles indicate the 12 carbon atoms belonging to cross-links. The MSD is calculated at 150 ps for a PNIPAM mass fraction of 30\% (left panel), 40\% (central panel), and 60\% (right panel). Errors within the symbol size.

concentration and exploring a wide range of temperatures, we probe PNIPAM and water dynamics in detail. We find that below $T_{d}$ both PNIPAM and water motions are characterized by an Arrhenius behavior, controlled by methyl rotations and hydrogen bonds, respectively. By monitoring the hydrogen bonding pattern, we find that long-lived PNIPAM-water bonds are primarily responsible for the observed water behavior at low temperatures. This is confirmed by the comparison with bulk water, which does not display the same features. $^{21}$ Our results clearly highlight the fact that the dynamical transition is a feature genuinely associated with water-macromolecule coupling.
In biological macromolecules, the protein dynamical transition is usually detected by monitoring the atomic mean squared displacements (MSD) through several experimental techniques. $^{1,22,23}$ The MSD can also be easily calculated as a function of time by molecular dynamics simulations. Its value at a given time, equal to that of a given experimental resolution, can then be monitored as a function of $T$. This is done in Figure 1B where the MSD of PNIPAM hydrogen atoms of a portion of microgel is reported for both long ( $1 \mathrm{~ns}$ ) and short (150 ps) times. On increasing temperature, a sudden promotion of large amplitude motions is visible in both cases, thus excluding the possibility that the observation of a 

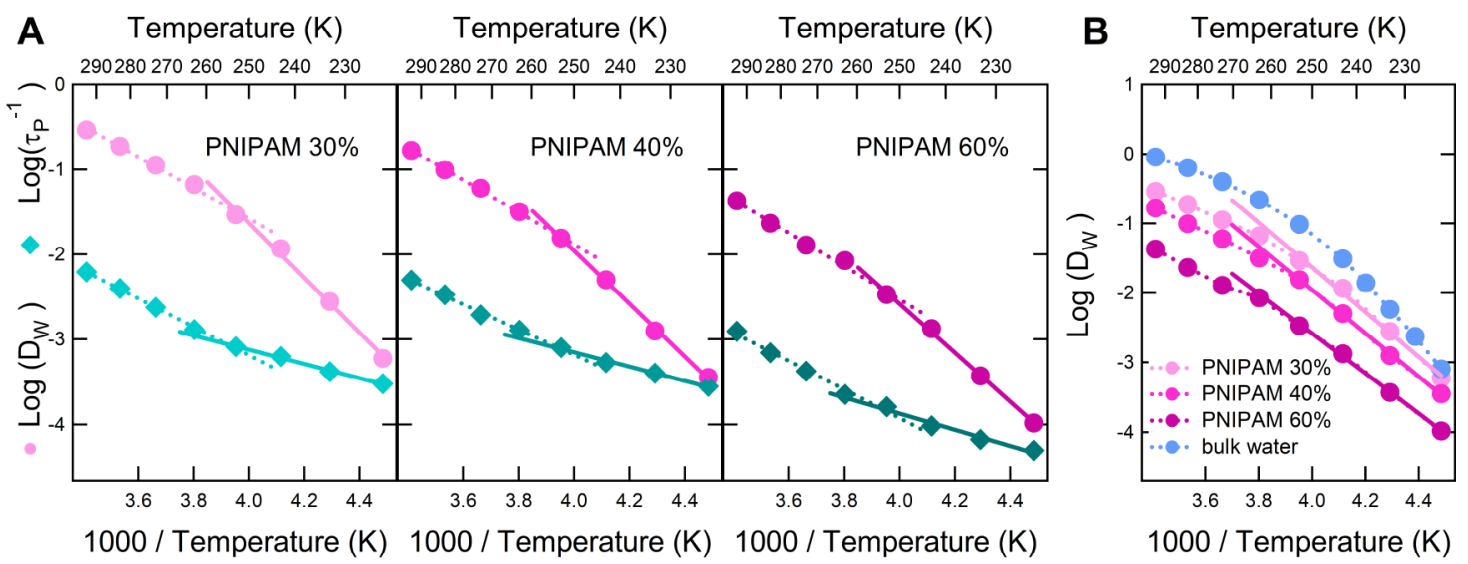

Figure 3. Arrhenius plots of (A) water diffusion coefficient $D_{w}$ (circles, in $\mathrm{cm}^{2} / \mathrm{s} \times 10^{5}$ ) and SISF relaxation times for PNIPAM hydrogen atoms $\tau_{p}$ (diamonds, in ps) calculated for PNIPAM mass fraction of 30\% (left panel), 40\% (central panel), and 60\% (right panel). (B) Water diffusion coefficient $\left(\right.$ in $\mathrm{cm}^{2} / \mathrm{s} \times 10^{5}$ ) calculated for bulk water and hydration water for different PNIPAM concentrations. In all panels, solid lines are Arrhenius fits to the data and dotted lines are guides to the eye.

transition is an artifact induced by the resolution limit of the accessible time or frequency window. ${ }^{24}$ In addition, the concentration dependence of the MSD reveals that the transition is more pronounced in more hydrated systems, in agreement with what has been found for proteins. ${ }^{14}$ This overall qualitative description of the molecular process does not allow full understanding of the microscopic interplay between water and the macromolecule originating this effect. Therefore, in the following, we analyze in detail the dynamical behavior of both PNIPAM and water.

First of all, it is important to exclude that the transition is due to an underlying structural change in the system. To this aim, we analyzed the structural alterations of the polymer matrix in the 200-290 K interval. Similarly to what is observed in proteins, ${ }^{1}$ no structural variations of the polymer network were found at $T_{d}$, neither as a whole, as shown by the temperature dependence of PNIPAM radius of gyration reported in Figure 2A, nor locally, as visible from the distributions of dihedral angles of the backbone and the temperature dependence of intramolecular hydrophilic and hydrophobic interactions reported in Figures S1 and S2, thus confirming the kinetic character of the transition.

The homogeneity of chemical composition in PNIPAM microgels allows us to identify a hierarchy of motion modes. The expected fastest motion is the torsion of side chain methyl groups, which is active at all the explored temperatures (Figure S3 and Table S1). Indeed by extending the explored temperature range down to $63 \mathrm{~K}$, an onset of anharmonicity consistent with the activation of methyl group rotation is detected at about $150 \mathrm{~K}$ (see Figure S4), in agreement with the behavior observed in biomacromolecules. ${ }^{8,25-27}$ We find that the average lifetime of a methyl rotational state is independent of the degree of hydration and follows an Arrhenius behavior (Figure 2B) for all investigated temperatures with an activation energy, $E_{a}^{\text {met }}$, of about $13.5 \mathrm{~kJ} \mathrm{~mol}^{-1}$ (Table S3). This value is similar to that reported for methyl groups in the hydrophobic core of proteins, in homomeric polypeptides and in bulk polymers. $^{25,28,29}$

Another class of motions in the polymer network can be ascribed to the rotation of the backbone dihedral angles. Figure 2C shows the evolution of the fraction of mobile backbone dihedral angles $x_{m}$ with $T$. Its behavior closely resembles that of the MSD behavior of PNIPAM (Figure 1B): the mobile backbone dihedral angles are very scarce below $T_{d}$, and an abrupt increase of their number occurs at and above $T_{d}$. The details of the backbone torsional dynamics are reported in the SI text (see Table S2). A further analysis of atomic motions has been carried out by calculating the MSD of the tertiary carbon atoms of the backbone. Figure 2D shows that the segmental dynamics of the polymer scaffold is quenched up to $\sim 250 \mathrm{~K}$ but is switched on at higher temperatures. In addition, while the dynamical behavior of PNIPAM is overall homogeneous below $T_{d}$, above this temperature we observe the onset of dynamical heterogeneities. Namely, the carbon atoms belonging to the junctions of the network (solid points in Figure 2D) develop lower local diffusivities, evidencing the role of the topological constraints on the dynamics. The comparison between different PNIPAM concentrations highlights the plasticizing effect of water, which results in a larger mobility for a higher degree of hydration. These results focus the principal role played by the backbone dynamics in the dynamical transition, in agreement with what observed in experimental studies on polypeptides. ${ }^{25,26}$ Moreover, the enhancement of anharmonic fluctuations at increasing water content (Figures $1 \mathrm{~B}$ and 2D) is similar to what observed for polypeptides in the presence of side chains. ${ }^{26}$

To monitor the PNIPAM dynamics as a function of $T$, we consider the self-intermediate scattering function (SISF) of PNIPAM hydrogen atoms (Table S4 and SI text), which is well described, at long times, by a stretched exponential with characteristic time $\tau_{p}$. The SISF is sensitive to the single particle dynamics at a specific wavevector $Q$. We focus on the value $Q=2.25 \AA^{-1}$, corresponding, in real space, to the position of the first maximum of the oxygen-oxygen structure factor in bulk water. ${ }^{30}$ In Figure $3 \mathrm{~A}$ an Arrhenius plot of $\tau_{p}$ is reported for three different PNIPAM concentrations, indicating a slowing down at the highest PNIPAM concentration, while the systems at $30 \%$ and $40 \%(\mathrm{w} / \mathrm{w})$ of polymer display similar values of $\tau_{p}$ (Table S3). We also report in Figure 3A the water translational self-diffusion coefficient $D_{w}$ as a function of $T$. The direct comparison between PNIPAM and water dynamics allows us to highlight several important features. First of all, we notice that both $\tau_{p}$ and $D_{w}$ display a change of behavior around $\sim 250 \mathrm{~K}$, that we identify as the dynamical transition. Analyzing the behaviors of $\tau_{p}$ and $D_{w}$ in detail, we notice that they are compatible with an Arrhenius dependence 

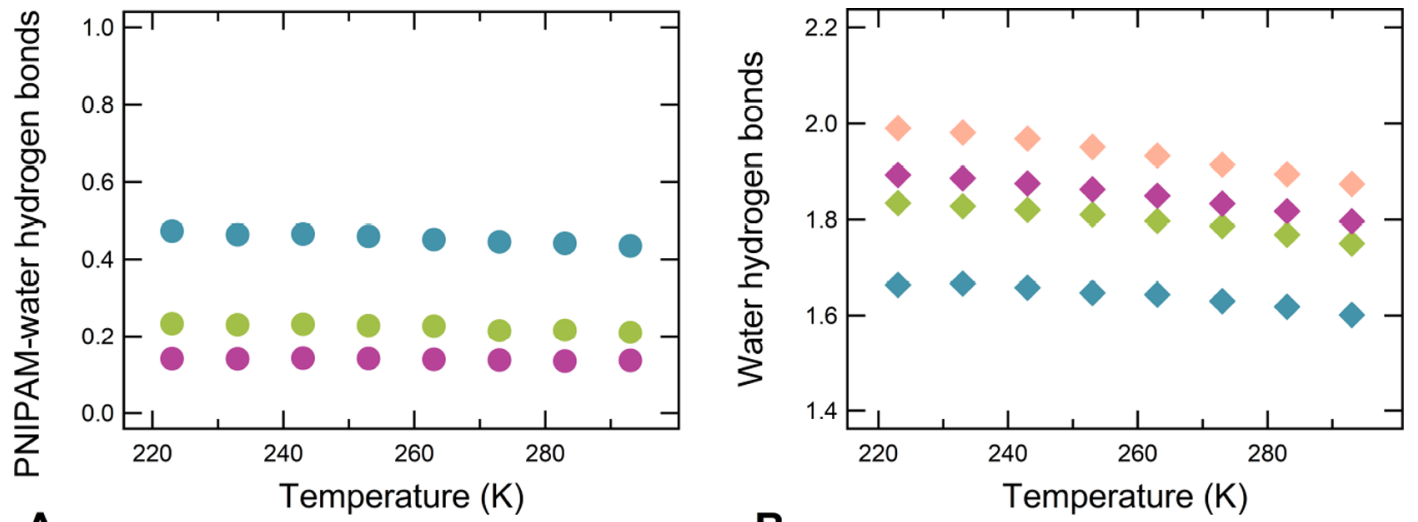

A

B
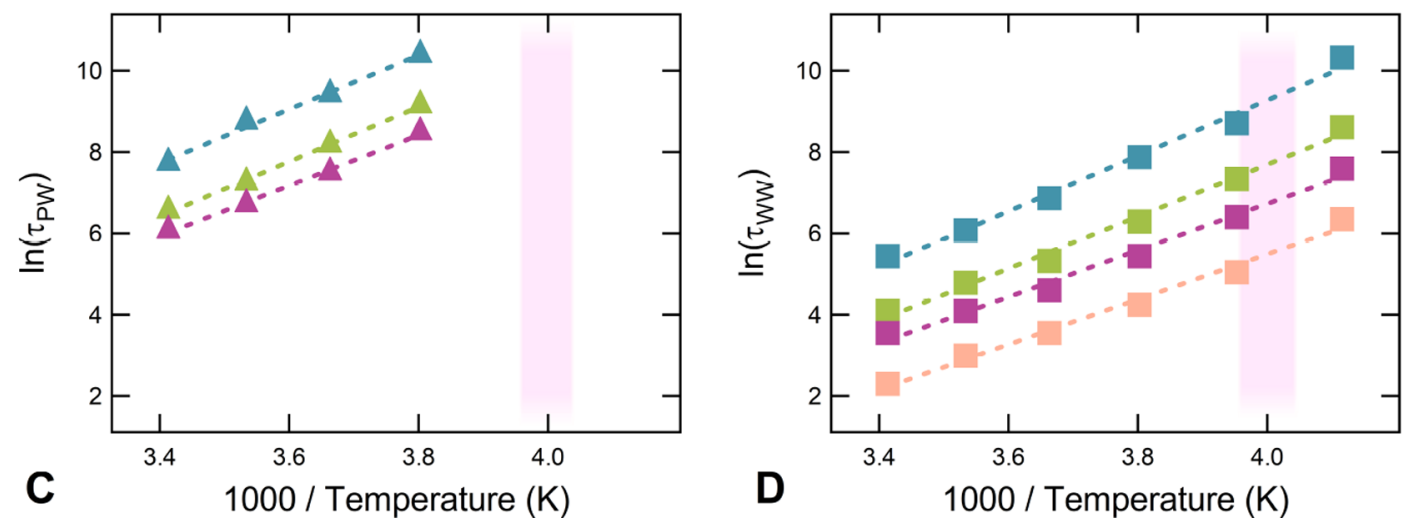

Figure 4. Temperature dependence of the average number of hydrogen bonds between PNIPAM and water (A) and between water molecules (B) per water molecule; Arrhenius plot of the average lifetime (in ps) of PNIPAM-water hydrogen bonds $\tau_{P W}(\mathrm{C})$ and of water-water hydrogen bond $\tau_{W W}(\mathrm{D})$. Results referring to PNIPAM mass fraction of $30 \%, 40 \%$, and $60 \mathrm{wt} \%$ are displayed in purple, green, and blue, respectively. Bulk water data are shown in pink. The pink frames highlight the region where the dynamical transition occurs. Errors within the symbol size.

both below and above $T_{d \cdot}{ }^{19}$ However, we will discuss later that only the low-temperature regime can be attributed to a true activation process. Instead, the high temperature data show a remarkably similar $T$-dependence for both $\tau_{p}$ and $D_{w}$ at all explored concentrations with a common apparent Arrhenius slope. We further notice that an inversion of the (putative) Arrhenius slope is detected between PNIPAM and water across $T_{d}$. In particular, for PNIPAM there is a crossover between a lower and a higher slope regime when temperature is increased above $T_{d}$, while water shows the opposite trend.

We recall that the number of hydration water molecules for PNIPAM is experimentally estimated to be $\sim 12 \pm 1$ per residue below the lower critical solution temperature. ${ }^{31,32}$ Therefore, for the three concentration values considered in this work, the water molecules can be entirely classified as hydration water (see also Experimental Section). Hence, it is instructive to compare the T-dependence of $D_{w}$ between hydration water and bulk water, as shown in Figure 3B. No clearly identifiable change at $T_{d}$ occurs for bulk water, suggesting the central role of PNIPAM-water coupling in the occurrence of the dynamical transition. Having identified the presence of a dynamical coupling between PNIPAM and water, we now ask the question whether a change of connectivity for the water molecules occurs at the transition. To this aim, we report in Figure 4A,B the temperature behavior of the number of PNIPAM-water and water-water hydrogen bonds (HBs), respectively. None of these observables display a sharp variation at $T_{d}$. We find an increasing number of PNIPAM-water HBs as a function of PNIPAM concentration and concomitantly a decrease of the number of water-water HBs, with no discontinuity with respect to the behavior of bulk water. This suggests that no increase of water structuring is originated in the surroundings of the hydrophobic groups. It is important to stress that the overall water structuring in microgel suspensions is always larger than for bulk water, with the total number of $\mathrm{HBs}$ (water-water and water-PNIPAM) increasing with polymer concentration (Figure S5). Thus, we can discard a structural origin of the transition in terms of $\mathrm{HB}$ connectivity.

We further monitor the characteristic lifetime of PNIPAMwater $\tau_{P W}$ and water-water $\tau_{W W}$ HB interactions. As shown in Figure $4 \mathrm{C}$ and $4 \mathrm{D}, \tau_{P W}$ and $\tau_{W W}$ both follow an Arrhenius behavior with similar values of activation energies of about 55 $\mathrm{kJ} \mathrm{mol}^{-1}$ (Table S3), irrespective of hydration level. We also note that the lifetime of PNIPAM-water HB is considerably longer than the lifetime of water-water $\mathrm{HB}$ and cannot be estimated below $T_{d}$ due to the finite simulation time. This finding is in agreement with previous results on the protein dynamical transition, ${ }^{33}$ which suggested that the lifetime of protein-water $\mathrm{HB}$ interactions diverges at $T_{d}$. The activation energy of $\mathrm{HB}$ is equal to that estimated from the Arrhenius dependence of $D_{w}$ for $T<T_{d}$ (see Table S3). Instead, the PNIPAM relaxation time $\tau_{p}$ has an activation energy of $\sim 16 \mathrm{~kJ}$ $\mathrm{mol}^{-1}$ for $T<T_{d}$ which is independent of the concentration and is comparable to that calculated for the rotation of the methyl groups (see Figure 2B). These findings suggest that below $T_{d}$ PNIPAM dynamics is dominated by methyl rotations, while the translational dynamics of water is 
controlled by its hydrogen bonding within the microgel environment.

For $T>T_{d}$, both observables $\tau_{p}$ and $D_{w}$ show a similar temperature dependence, that could be interpreted as an Arrhenius regime, ${ }^{19}$ providing activation energies very close to each other, i.e., $\sim 30 \pm 2 \mathrm{~kJ} \mathrm{~mol}^{-1}$ for $\tau_{p}$ and $\sim 36 \pm 2 \mathrm{~kJ} \mathrm{~mol}^{-1}$ for $D_{w}$. However, these values cannot be attributed to any specific structural rearrangement, indicating that this apparent Arrhenius behavior ${ }^{34}$ may result from the superposition of different contributions. The fact that the apparent activation energy is higher than the methyl rotation one and lower than the one corresponding to $\mathrm{HB}$ lifetime gives rise to a "slope" inversion of PNIPAM and water dynamics, as observed in the data of Figure 3, similarly to hydrated lysozyme results. ${ }^{35}$ Hence, above the dynamical transition temperature, we cannot distinguish a dominant specific molecular motion, but rather we only detect a correlation between polymer segmental dynamics and diffusive motion of bound water. This dynamical interplay can only be active when the PNIPAM-water $\mathrm{HB}$ lifetime is sufficiently short that the exchange of hydrogen bonded water molecules is still effective ${ }^{33}$ and was recently confirmed in the water-soluble states of a PNIPAM linear chain. $^{36}$

Differently, the dynamical transition clearly marks the onset, below $T_{d}$, of distinct dominant motions, respectively, for PNIPAM and water. The comparison with bulk water further allows us to identify that it is primarily the hydrogen bond pattern of water within the microgel, dictated by the slowest PNIPAM-water $\mathrm{HB}$, that dominates the low- $T$ water dynamics. The $T$-dependence of $D_{w}$ for bulk water is further analyzed in the Supporting Information, where it is shown that at high temperatures the data are well described by a powerlaw decay (see Figure S6 Supporting Information) in agreement with previous studies. $^{21,35}$ For low $T$, also bulk water follows an Arrhenius dependence but with a much higher activation energy $\left(\sim 80 \mathrm{~kJ} \mathrm{~mol}^{-1}\right)$, indicating a different mechanism than hydrogen bonding controlling its dynamics, even if the number of water-water $\mathrm{HB}$ increases as $T$ decreases. This leads us to exclude a direct influence of waterwater hydrogen bonding on the dynamical transition. Instead, we can clearly isolate the contribution of PNIPAM-water hydrogen bonding, which is slower than water-water HB by roughly 2 orders of magnitude at each $T$ for the same PNIPAM concentration (see Figure 4C,D). Therefore, PNIPAM-water hydrogen bonding must be the trigger of the Arrhenius dependence below $T_{d}$ and thus the microscopic mechanism responsible for the occurrence of the dynamical transition.

It is also interesting to discuss the relatively large value of $T_{d}$ for PNIPAM microgels with respect to protein systems. The average number of macromolecule-water hydrogen bonds formed was found to be 1.2 and 1.1 for lysozyme and plastocyanin per protein residue, respectively, ${ }^{18,37}$ while for PNIPAM-water hydrogen bonds we find $\sim 2 .{ }^{38}$ Thus, the different macromolecule-water interaction may play a primary role in determining the value of $T_{d}$. We also note that the value of $T_{d}$ was also found to depend on the hydration level $h^{4,39,40}$ ( $h=\mathrm{g}$ water/g protein), with lower values found for higher water content. However, such a variation appears to be significant at low $h{ }^{39}$ tending to saturate at large water content. We stress that the PNIPAM mass fractions that we have explored correspond to water content $h=2.33,1.5$, and 0.67 for $30 \%, 40 \%$, and $60 \%$, respectively, thus to a completely different water content region with respect to all previous studies.

In conclusion, the present results provide a microscopic description of the origin of the "protein-like" dynamical transition observed in microgels. The correlation between the information extracted from the analysis of PNIPAM relaxations times, water self-diffusion coefficients, and hydrogen bonding interactions allowed us to identify the molecular processes which control the dynamics of both PNIPAM and water below the dynamical transition temperature. In particular, we found that below $T_{d}$ PNIPAM dynamics is governed by the rotation of the methyl groups belonging to the side chains and that a sudden increase of the polymer segmental dynamics occurs at $T_{d}$. On the other hand, hydrogen bonding interactions determine water dynamics below $T_{d}$. By comparing the low temperature behavior of water in the microgels suspensions to that of bulk water, we also found that the hydrogen bonding interactions between PNIPAM and water play the primary role in determining water dynamics below $T_{d}$. Altogether these findings support the idea that the macromolecule-water coupling is the driving ingredient of the dynamical transition. Thus, such phenomenology should be rather general, taking place in all hydrated macromolecular systems which are able, at the same time, to efficiently confine water in order to avoid ice formation and to couple it via hydrogen bonding. These findings also rule out the possibility to observe a dynamical transition in dry systems, where similar findings ${ }^{41}$ must then be ascribed to a different molecular mechanism.

\section{EXPERIMENTAL SECTION}

PNIPAM microgel is modeled as an isotropic network composed of 12 atactic chains connected by 6 bis(acrylamide) cross-links. Periodic images of the network are covalently bonded to mimic the 3-D percolation of the microgel. This model has a monomer/cross-linker ratio that, given the heterogeneous structure of PNIPAM microgels, describes a region of the particle close to the core-shell boundary, and it quantitatively reproduces neutron scattering results. ${ }^{19}$ Three PNIPAM mass fractions of 30,40 , and $60 \%(\mathrm{w} / \mathrm{w})$, corresponding to hydration levels of 14,9 , and 4 water molecules per PNIPAM residue, respectively, were investigated. All-atom MD simulations are performed in the range between 293 and $223 \mathrm{~K}$. At each $T$, trajectory data are collected for $\sim 0.5 \mu \mathrm{s}$. We adopt the OPLS-AA force field ${ }^{42}$ with the implementation by Siu et al. ${ }^{43}$ for PNIPAM and the Tip4p/ICE model $^{44}$ for water. MD simulations are also carried out for a cubic box containing 1782 Tip4p/ICE water molecules following a similar procedure. Additional details are reported in Sections S7-S9 of the SI Text.

\section{ASSOCIATED CONTENT}

\section{Supporting Information}

The Supporting Information is available free of charge on the ACS Publications website at DOI: 10.1021/acs.jpclett.9b00190.

Sections presenting study of PNIPAM microgel structural rearrangements, torsional dynamics, activation energies, self-intermediate scattering functions (fitting procedure), water hydrogen bonding, bulk water diffusion coefficient, in silico model of PNIPAM microgel, simulation protocol, and data analysis (PDF) 


\section{AUTHOR INFORMATION}

\section{Corresponding Authors}

*(E.C.) E-mail: ester.chiessi@uniroma2.it.

*(E.Z.) E-mail: emanuela.zaccarelli@cnr.it.

\section{ORCID}

Letizia Tavagnacco: 0000-0002-3492-7766

Ester Chiessi: 0000-0001-7529-2755

Emanuela Zaccarelli: 0000-0003-0032-8906

Notes

The authors declare no competing financial interest.

\section{ACKNOWLEDGMENTS}

L.T., E.C., and E.Z. acknowledge support from the European Research Council (ERC Consolidator Grant 681597, MIMIC) and CINECA-ISCRA for HPC resources.

\section{REFERENCES}

(1) Doster, W.; Cusack, S.; Petry, W. Dynamical transition of myoglobin revealed by inelastic neutron scattering. Nature 1989, 337, 754-756.

(2) Rasmussen, B. F.; Stock, A. M.; Ringe, D.; Petsko, G. A. Crystalline ribonuclease A loses function below the dynamical transition at $220 \mathrm{~K}$. Nature 1992, 357, 423.

(3) Markelz, A. G.; Knab, J. R.; Chen, J. Y.; He, Y. Protein dynamical transition in terahertz dielectric response. Chem. Phys. Lett. 2007, 442, 413-417.

(4) Capaccioli, S.; Ngai, K. L.; Ancherbak, S.; Paciaroni, A. Evidence of Coexistence of Change of Caged Dynamics at Tg and the Dynamic Transition at Td in Solvated Proteins. J. Phys. Chem. B 2012, 116, $1745-1757$

(5) Caliskan, G.; Briber, R. M.; Thirumalai, D.; Garcia-Sakai, V.; Woodson, S. A.; Sokolov, A. P. Dynamic Transition in tRNA is Solvent Induced. J. Am. Chem. Soc. 2006, 128, 32-33.

(6) Cornicchi, E.; Capponi, S.; Marconi, M.; Onori, G.; Paciaroni, A. Temperature dependence of fast fluctuations in single- and doublestranded DNA molecules: a neutron scattering investigation. Philos. Mag. 2007, 87, 509-515.

(7) Peters, J.; Marion, J.; Natali, F.; Kats, E.; Bicout, D. J. The Dynamical Transition of Lipid Multilamellar Bilayers as a Matter of Cooperativity. J. Phys. Chem. B 2017, 121, 6860-6868.

(8) Roh, J. H.; Novikov, V. N.; Gregory, R. B.; Curtis, J. E.; Chowdhuri, Z.; Sokolov, A. P. Onsets of Anharmonicity in Protein Dynamics. Phys. Rev. Lett. 2005, 95, 038101.

(9) He, Y.; Ku, P. I.; Knab, J. R.; Chen, J. Y.; Markelz, A. G. Protein Dynamical Transition Does Not Require Protein Structure. Phys. Rev. Lett. 2008, 101, 178103.

(10) Schirò, G.; Fichou, Y.; Gallat, F.-X.; Wood, K.; Gabel, F.; Moulin, M.; Härtlein, M.; Heyden, M.; Colletier, J.-P.; Orecchini, A.; et al. Translational diffusion of hydration water correlates with functional motions in folded and intrinsically disordered proteins. Nat. Commun. 2015, 6, 6490.

(11) Henzler-Wildman, K.; Kern, D. Dynamic personalities of proteins. Nature 2007, 450, 964

(12) Dioumaev, A. K.; Lanyi, J. K. Bacteriorhodopsin photocycle at cryogenic temperatures reveals distributed barriers of conformational substates. Proc. Natl. Acad. Sci. U. S. A. 2007, 104, 9621-9626.

(13) Henzler-Wildman, K. A.; Lei, M.; Thai, V.; Kerns, S. J.; Karplus, M.; Kern, D. A hierarchy of timescales in protein dynamics is linked to enzyme catalysis. Nature 2007, 450, 913.

(14) Roh, J.; Curtis, J.; Azzam, S.; Novikov, V.; Peral, I.; Chowdhuri, Z.; Gregory, R.; Sokolov, A. Influence of hydration on the dynamics of lysozyme. Biophys. J. 2006, 91, 2573-2588.

(15) Chen, S.-H.; Liu, L.; Fratini, E.; Baglioni, P.; Faraone, A.; Mamontov, E. Observation of fragile-to-strong dynamic crossover in protein hydration water. Proc. Natl. Acad. Sci. U. S. A. 2006, 103, 9012-9016.
(16) Frauenfelder, H.; Chen, G.; Berendzen, J.; Fenimore, P. W.; Jansson, H.; McMahon, B. H.; Stroe, I. R.; Swenson, J.; Young, R. D. A unified model of protein dynamics. Proc. Natl. Acad. Sci. U. S. A 2009, 106, 5129-5134.

(17) Khodadadi, S.; Roh, J.; Kisliuk, A.; Mamontov, E.; Tyagi, M.; Woodson, S.; Briber, R.; Sokolov, A. Dynamics of Biological Macromolecules: Not a Simple Slaving by Hydration Water. Biophys. J. 2010, 98, 1321-1326.

(18) Nandi, P. K.; English, N. J.; Futera, Z.; Benedetto, A. Hydrogen-bond dynamics at the bio-water interface in hydrated proteins: a molecular-dynamics study. Phys. Chem. Chem. Phys. 2017, 19, 318-329.

(19) Zanatta, M.; Tavagnacco, L.; Buratti, E.; Bertoldo, M.; Natali, F.; Chiessi, E.; Orecchini, A.; Zaccarelli, E. Evidence of a lowtemperature dynamical transition in concentrated microgels. Science Advances 2018, 4, No. eaat5895.

(20) The estimate of the water accessible surface area in a PNIPAM microgel at a concentration of $60 \%(\mathrm{w} / \mathrm{w})$ and in a hydrated lysozyme sample at the concentration of $67 \%(\mathrm{w} / \mathrm{w})$ gives the values of $2.6 \times$ $10^{6}$ and $2.0 \times 10^{6} \mathrm{~m}^{2} \mathrm{~kg}^{-1}$, respectively.

(21) De Marzio, M.; Camisasca, G.; Rovere, M.; Gallo, P. Mode coupling theory and fragile to strong transition in supercooled TIP4P/2005 water. J. Chem. Phys. 2016, 144, 074503.

(22) Parak, F.; Knapp, E.; Kucheida, D. Protein dynamics: Mössbauer spectroscopy on deoxymyoglobin crystals. J. Mol. Biol. 1982, 161, 177-194.

(23) Melchers, B.; Knapp, E.; Parak, F.; Cordone, L.; Cupane, A.; Leone, M. Structural fluctuations of myoglobin from normal-modes, Mössbauer, Raman, and absorption spectroscopy. Biophys. J. 1996, 70, 2092-2099.

(24) Khodadadi, S.; Pawlus, S.; Roh, J. H.; Garcia Sakai, V.; Mamontov, E.; Sokolov, A. P. The origin of the dynamic transition in proteins. J. Chem. Phys. 2008, 128, 195106.

(25) Schiró, G.; Caronna, C.; Natali, F.; Cupane, A. Direct evidence of the amino acid side chain and backbone contributions to protein anharmonicity. J. Am. Chem. Soc. 2010, 132, 1371-1376.

(26) Schiró, G.; Caronna, C.; Natali, F.; Cupane, A. Molecular origin and hydration dependence of protein anharmonicity: an elastic neutron scattering study. Phys. Chem. Chem. Phys. 2010, 12, 1021510220 .

(27) Telling, M. T. F.; Neylon, C.; Clifton, L.; Howells, S.; van Eijck, L.; Sakai, V. G. Thermal motion in the multi-subunit protein, apoferritin, as probed by high energy resolution neutron spectroscopy. Soft Matter 2011, 7, 6934-6941.

(28) Xue, Y.; Pavlova, M. S.; Ryabov, Y. E.; Reif, B.; Skrynnikov, N. R. Methyl Rotation Barriers in Proteins from 2H Relaxation Data. Implications for Protein Structure. J. Am. Chem. Soc. 2007, 129, $6827-6838$

(29) Ahumada, O.; Theodorou, D. N.; Triolo, A.; Arrighi, V.; Karatasos, C.; Ryckaert, J.-P. Segmental Dynamics of Atactic Polypropylene As Revealed by Molecular Simulations and Quasielastic Neutron Scattering. Macromolecules 2002, 35, 7110-7124.

(30) Sciortino, F.; Gallo, P.; Tartaglia, P.; Chen, S. H. Supercooled water and the kinetic glass transition. Phys. Rev. E: Stat. Phys., Plasmas, Fluids, Relat. Interdiscip. Top. 1996, 54, 6331-6343.

(31) Ono, Y.; Shikata, T. Hydration and Dynamic Behavior of Poly(N-isopropylacrylamide)s in Aqueous Solution: A Sharp Phase Transition at the Lower Critical Solution Temperature. J. Am. Chem. Soc. 2006, 128, 10030-10031.

(32) Satokawa, Y.; Shikata, T.; Tanaka, F.; Qiu, X.-p.; Winnik, F. M. Hydration and Dynamic Behavior of a Cyclic Poly(N-isopropylacrylamide) in Aqueous Solution: Effects of the Polymer Chain Topology. Macromolecules 2009, 42, 1400-1403.

(33) Tarek, M.; Tobias, D. J. Role of Protein-Water Hydrogen Bond Dynamics in the Protein Dynamical Transition. Phys. Rev. Lett. 2002, 88,138101

(34) Kanaya, T.; Kawaguchi, T.; Kaji, K. Local Dynamics of Some Bulk Polymers above Tg As Seen by Quasielastic Neutron Scattering. Macromolecules 1999, 32, 1672-1678. 
(35) Camisasca, G.; De Marzio, M.; Corradini, D.; Gallo, P. Two structural relaxations in protein hydration water and their dynamic crossovers. J. Chem. Phys. 2016, 145, 044503.

(36) Tavagnacco, L.; Zaccarelli, E.; Chiessi, E. On the molecular origin of the cooperative coil-to-globule transition of poly $(\mathrm{N}$ isopropylacrylamide) in water. Phys. Chem. Chem. Phys. 2018, 20, 9997-10010.

(37) Arcangeli, C.; Bizzarri, A. R.; Cannistraro, S. Role of interfacial water in the molecular dynamics-simulated dynamical transition of plastocyanin. Chem. Phys. Lett. 1998, 291, 7-14.

(38) For PNIPAM $40 \%$ mass fraction, the number of PNIPAMwater hydrogen bonds normalized to the number of PNIPAM residues is obtained by multiplying the value reported in Figure 4A to the number of water molecules (1676) and dividing it by the number of PNIPAM residues (180).

(39) Paciaroni, A.; Cinelli, S.; Cornicchi, E.; De Francesco, A.; Onori, G. Fast fluctuations in protein powders: the role of hydration. Chem. Phys. Lett. 2005, 410, 400-403.

(40) Kim, S. B.; Gupta, D. R.; Debenedetti, P. G. Computational investigation of dynamical transitions in Trp-cage miniprotein powders. Sci. Rep. 2016, 6, 25612.

(41) Liu, Z.; Huang, J.; Tyagi, M.; O’Neill, H.; Zhang, Q.; Mamontov, E.; Jain, N.; Wang, Y.; Zhang, J.; Smith, J. C.; et al. Dynamical Transition of Collective Motions in Dry Proteins. Phys. Rev. Lett. 2017, 119, 048101.

(42) Jorgensen, W. L.; Maxwell, D. S.; Tirado-Rives, J. Development and Testing of the OPLS All-Atom Force Field on Conformational Energetics and Properties of Organic Liquids. J. Am. Chem. Soc. 1996, $118,11225-11236$.

(43) Siu, S. W. I.; Pluhackova, K.; Böckmann, R. A. Optimization of the OPLS-AA Force Field for Long Hydrocarbons. J. Chem. Theory Comput. 2012, 8, 1459-1470.

(44) Abascal, J. L. F.; Sanz, E.; García Fernández, R. G.; Vega, C. A potential model for the study of ices and amorphous water: TIP4P/ Ice. J. Chem. Phys. 2005, 122, 234511. 